\title{
EL PROBLEMA FREGE-GEACH
}

José Juan Moreso*

P.T. Geach, en un trabajo en el cual a partir de ciertos pasajes de G. Frege, llamaba la atención sobre la posibilidad de que una proposición pueda aparecer en el discurso como aseverada o como no aseverada, por ejemplo: supuesta o imaginada, y seguir siendo la misma proposición, ${ }^{2}$ elaboró un argumento contra las concepciones no-cognoscitivistas de la ética. Un argumento con arreglo al cual dichas concepciones de la ética no son capaces de dar cuenta del comportamiento de los juicios morales cuando aparecen en contextos gramaticalmente complejos, en contextos encapsulados (embedded contexts). Desde entonces en la literatura se lo conoce como el problema Frege-Geach o, también, como el problema de los contextos encapsulados. Estas son las palabras de Geach: ${ }^{3}$

The theory that to call a kind of act 'bad' is not to describe but to condemn it is open to similar objections. Let us consider this piece of moral reasoning:

If doing a thing is bad, getting your little brother to do it is bad.

Tormenting the cat is bad.

Ergo, getting your little brother to torment the cat is bad.

\footnotetext{
* Universitat Pompeu Fabra, Barcelona.

${ }^{1}$ Este trabajo fue presentado en el I Foro Analítico de Filosofía del Derecho, realizado en la Universidad de Murcia en febrero del 2002.

${ }^{2}$ P.T. Geach, ‘Assertion', Philosophical Review, 74 (1965): 449-465.

${ }^{3}$ P.T. Geach, ‘Assertion', supra en n. 1, pp. 463-464.
} 
The whole nerve of the reasoning is that 'bad' should mean exactly the same at all four occurrences -should not, for example, shift from an evaluative to a descriptive or conventional or inverted-commas use. But in the major premise the speaker (a father, let us suppose) is certainly not uttering acts of condemning: one could hardly take him to be condemning just doing a thing.

Como es sabido, las concepciones no-cognoscitivistas de la ética (emotivistas, prescriptivistas o, como parece preferirse ahora, expresivistas $)^{4}$ sostienen que los juicios morales no son aptos para la verdad y la falsedad, puesto que no representan el mundo, no son candidatos a contenidos de nuestras creencias, sino que expresan nuestras actitudes de aprobación y desaprobación hacia determinadas acciones o estados de cosas. Pues bien, el argumento de los contextos encapsulados plantea un aparente dilema a dichas concepciones: o bien argumentos aparentemente válidos como el de Geach, una instancia del modus ponens, no lo son porque el antecedente de su primera premisa ('Si hacer una cosa está mal') no es en ningún plausible sentido la expresión de una actitud y entonces 'bad' tiene un sentido en la primera premisa que no coincide con el sentido de 'bad' en la segunda premisa y en la conclusión, o bien los juicios morales no son la expresión de actitudes, sino que expresan contenidos proposicionales y, por esta razón, pueden figurar

${ }^{4}$ Las obras de referencia del emotivismo son A.J. Ayer, Language, Truth and Logic (London: Gollancz, 1936) y Charles Stevenson, Ethics and Language (New Haven: Yale University Press, 1944); del prescriptivismo R.M. Hare, The Language of Morals (Oxford: Oxford University Press, 1952); las versiones recientes del expresivismo están representadas, con especial atención al problema Frege-Geach, por Simon Blackburn, Spreading the Word (Oxford: Oxford University Press, 1984) y 'Attitudes and Contents' en Essays in Quasi-Realism (Oxford: Oxford University Press, 1993), 182-197 y Allan Gibbard, Wise Choices, Apt Feelings (Oxford: Oxford University Press, 1990). 
como antecedentes de condicionales, o componentes de disyunciones o elementos de otros contextos encapsulados.

Los modernos defensores del expresivismo han intentado ofrecer alguna respuesta a Geach, consistente en mostrar cómo su razonamiento puede ser válido sin abandonar la idea de que los juicios morales son primordialmente expresiones de actitudes y no son, por lo tanto, aptos para la verdad. ${ }^{5}$ Blackburn ha desarrollado una sofisticada lógica de actitudes que trata de mostrar cómo es posible encapsular los juicios morales manteniendo su naturaleza semántica. ${ }^{6}$ Gibbard ha elegido una estrategia indirecta, consistente en adoptar una lectura descriptiva de los operadores deónticos que aparecen en los razonamientos con juicios morales encapsulados. ${ }^{7}$ No voy a discutir aquí con detalle ninguna de ambas propuestas, sino que voy a presentar de forma algo esquemática una estrategia de solución para el problema Frege-Geach que no coincide con ninguna de las comentadas, aunque tiene algunos puntos de contacto con la estrategia de Gibbard.

Tal vez la discusión de este problema, y de otros conectados con él, en filosofía moral se beneficiaría si tomaran en cuenta dos ideas que si

${ }^{5}$ A ello respondía, parcialmente, la distinción de Hare de ya no dos elementos sino tres en su teoría del significado: frástico, trópico y néustico. Vd. R.M. Hare, 'Meaning and Speech Acts' en Practical Inferences, (London: MacMillan, 1971), 74-93.

${ }^{6}$ Simon Blackburn, Spreading the Word, supra en n. 3, cap. 6 y 'Attitudes and Contents', supra n. 3, 182-197. Véase la discusión suscitada en Bob Hale, 'The Compleat Projectivist' Philosophical Review, 36 (1986): 85-84 y 'Can There Be a Logic of Attitudes?' en John Haldane, Crispin Wright (eds.), Reality, Representation and Projection (Oxford: Oxford University Press, 1993), 337-365; Nick Zangwill, 'Moral Modus Ponens', Ratio 2 (1992): 177-193; Nicholas Unwin, 'Quasi-Realism, Negation and the Frege-Geach Problem', The Philosophical Quarterly, 49 (1999): 337-352.

${ }^{7}$ Allan Gibbard, Wise Choices, Apt Feelings, supra en n. 3, cap. 5. Puede verse alguna literatura crítica sobre este intento en Simon Blackburn, 'Wise Feelings, Apt Reading', Ethics, 102 (1992): 342-356; Paul Horwich, 'Gibbard's Theory of Norms', Philosophy and Public Affairs, 22 (1993): 67-78 y Nicholas Unwin, 'Norms and Negation: A Problem for Gibbard's Logic', The Philosophical Quarterly, 51 (2001): 60-75. 
bien no son habituales en la literatura ética, son usuales en la literatura iusfilosófica. Me refiero a las dos ideas siguientes:

(i) La ambigüedad sistemática de los enunciados deónticos. ${ }^{8} \mathrm{Un}$ enunciado como 'Aparcar en doble fila en el Paseo de Gracia está prohibido' es ambiguo, puesto que tanto puede ser la expresión de una norma genuina, dictada por una autoridad (municipal, por ejemplo), o puede ser la expresión de una proposición normativa, una aserción acerca de la pertenencia a determinado conjunto de normas de una norma que prohibe aparcar en doble fila en el Paseo de Gracia. ${ }^{9}$ Mientras la primera no es susceptible de verdad o falsedad puesto que es una prescripción dirigida a influir en el comportamiento de sus destinatarios, la segunda es apta para la verdad, puesto que trata de representar el estado de cosas tal que en determinado conjunto normativo hay una norma con determinado contenido. Pues bien, de una manera similar una oración como 'Tormenting the cat is bad' es sistemáticamente ambigua, puesto que puede ser la expresión de una actitud o la descripción de la pertenencia a determinado conjunto de normas morales de una norma que prohibe torturar a los gatos, junto con la definición de que aquello prohibido por las normas morales es 'bad'. ${ }^{10}$

(ii) La idea conforme a la cual en los conjuntos normativos no suele haber únicamente normas, en el sentido de significados de oraciones

${ }^{8}$ La expresión, como es sabido, procede de von Wright. Georg Henrik von Wright, Norm and Action. A Logical Enquiry, (London: Routledge \& Kegan Paul, 1963), p. 105.

${ }^{9}$ Esta forma de presentar la distinción es, como resulta obvio, la de Carlos E. Alchourrón (por ejemplo en 'Logic of Norms and Logic of Normative Propositions', Logique et Analyse, 12 (1969): 242-268) y Eugenio Bulygin (puede verse 'Norms, Normative Propositions, and Legal Statements' en G. Floistad (ed.), Contemporary Philosophy. A New Survey. Vol. 3: Philosphy of Action (The Hague: Martinus Nijhoff, 1981), 107-125.

${ }^{10}$ Aquí es irrelevante, para el argumento de Geach, que dicho conjunto de normas morales sea el conjunto de normas morales aceptado por determinada persona, o el conjunto de normas morales que toda persona racional aceptaría, o cualquiera otra forma de delimitar un conjunto de normas morales. 
prescriptivas, sino también otro tipo de reglas, que en la literatura se conocen como reglas determinativas, conceptuales, constitutivas o cualificatorias. ${ }^{11}$

De hecho, estas dos ideas junto con la forma en que nuestros códigos penales suelen castigar la inducción a cometer delitos, puede iluminar el comportamiento del razonamiento de Geach. La sugerencia es la siguiente: 'es un delito' se comporta como 'is bad', es decir, usamos estos términos jurídicos (como en una ocasión sostuvo R. Carnap) ${ }^{12}$ de un modo 'transposed', decir que $x$ es un delito equivale a decir que $x$ se encuentra en la lista de delitos de nuestro código penal, de forma similar decir de un comportamiento $x$ que is bad, equivale a decir que es un comportamiento prohibido por una norma moral. Con estas ideas, podemos contemplar estos dos argumentos similares:

R1

(1) $\forall \mathrm{x}$ ( $\mathrm{x}$ es un delito $\rightarrow$ inducir a cometer $\mathrm{x}$ es un delito)

(2) El homicidio es un delito

Ergo, inducir a cometer homicidio es un delito

R2

(1) $\forall x$ (x es un comportamiento moralmente incorrecto $\rightarrow$ inducir a cometer $\mathrm{x}$ es un comportamiento moralmente incorrecto)

(2) Torturar a los gatos es un comportamiento moralmente incorrecto

\footnotetext{
${ }^{11}$ Entre las abundantes referencias pueden verse las siguientes: Georg Henrik von Wright, Norm and Action, supra n. 7, John Searle, Speech Acts (Cambridge: Cambridge University Press, 1971), Carlos E. Alchourrón, Eugenio Bulygin, 'Definiciones y normas' en El lenguaje del Derecho. Homenaje a G.R. Carrió (Buenos Aires: Abeledo-Perrot, 1981), 11-42, Rafael Hernández Marín, El Derecho como dogma (Madrid: Tecnos, 1984) y Teoría general del Derecho y de la ciencia jurídica (Barcelona: PPU, 1989), Manuel Atienza y Juan Ruiz Manero, Las piezas del Derecho (Barcelona. Ariel, 1996).

${ }^{12}$ Rudolf Carnap, The Logical Syntax of Language (London: Routledge \& Kegan Paul, 1971), p. 308.
} 
Ergo, inducir a torturar a los gatos es un comportamiento moralmente incorrecto

R1 y R2 son razonamientos lógicamente válidos, instancias del modus ponens, pero no plantean problema alguno al no-cognoscitivismo ético, puesto que R2 no es un razonamiento con juicios morales genuinos, sino que es un razonamiento acerca de algún conjunto de normas morales. En concreto, en R2, la verdad de (2) depende de que el sistema moral de referencia contenga una norma moral que prohiba torturar a los gatos y una definición (en el propio sistema, o realizada por el que lo describe) con arreglo a la cual los comportamientos moralmente prohibidos son moralmente incorrectos. La verdad de (1) depende de que el conjunto de normas morales contenga alguna regla definitoria al respecto de la inducción a cometer comportamientos prohibidos como podría ser la siguiente:

(RI) $\forall \mathrm{x} \forall \mathrm{y}$ ( $\mathrm{x}$ es una persona $\wedge \mathrm{y}$ es un comportamiento $\wedge \mathrm{x}$ es inductor de $\mathrm{y} \rightarrow \mathrm{x}$ es autor de $\mathrm{y})^{13}$

Para sostener que R1 y R2 son lógicamente válidos, no es precisa ninguna lógica de actitudes, ni lógica de normas ni nada por el estilo, basta con la lógica clásica, ahora bien el uso de 'moralmente incorrecto' en las premisas y la conclusión de R2, como el uso de ‘delito' en las premisas y conclusión de R1, no es un uso prescriptivo o expresivo, sino descriptivo.

Alguien podría replicar, sin embargo, que lo anterior concede la razón a Geach en un punto: si los juicios morales fueran expresiones de actitudes no podrían ser encapsulados. Ahora bien, es posible que los juicios morales, cuando son comprendidos como expresiones de actitudes no puedan ser

\footnotetext{
${ }^{13}$ De una forma similar a ésta, mediante definiciones, consigue el resultado de castigar la inducción el código penal español. Véanse sus arts. 27 y 28. Artículo 27: 'Son responsables criminalmente de los delitos y faltas los autores y los cómplices'. Artículo 28: 'Son autores quienes realizan el hecho por sí solos, conjuntamente o por medio de otro del que se sirven como instrumento. También serán considerados autores: a) Los que inducen directamente a otro u otros a ejecutarlo; b) Los que cooperan a su ejecución con un acto sin el cual no se habría efectuado'.
} 
encapsulados y, sin embargo, puedan serlo, cuando son comprendidos de manera similar a las proposiciones normativas. Esto es, es posible asumir el no-cognoscitivismo ético, conforme al cual los juicios morales son similares a prescripciones y asumir la validez de razonamientos como R2, porque los juicios morales contenidos en él no son genuinos, sino que son aserciones acerca de la pertenencia de determinadas normas morales a ciertos conjuntos normativos.

No obstante, para los que no somos escépticos acerca de la posibilidad de las relaciones lógicas entre normas, existe todavía la posibilidad de contemplar razonamientos como el de Geach como instancias de un modus ponens genuinamente normativo. Prestemos atención a los dos siguientes razonamientos:

R'1

(1) $\forall x$ (x es un comportamiento prohibido por una norma penal $\rightarrow$ Prohibido [inducir a cometer $\mathrm{x}$ ])

(2) El homicidio está prohibido por una norma penal

Ergo, Prohibido [inducir a cometer homicidio]

R'2

(1) $\forall x$ (x es un comportamiento prohibido por una norma moral $\rightarrow$ Prohibido [inducir a cometer $\mathrm{x}$ ])

(2) Torturar a los gatos es un comportamiento prohibido por una norma moral

Ergo, Prohibido [inducir a torturar a los gatos]

R'1 y R'2 son razonamientos lógicamente válidos de lógica de normas, si aceptamos la validez de un esquema según el cual, de ' $\mathrm{p} \rightarrow$ Oq' y 'p' se deriva 'Oq'. Yo no veo inconveniente en aceptar este esquema, pero presupone algunas asunciones que trataré de explicitar:

(i) Presupone que ' $\mathrm{p} \rightarrow$ Oq' es una forma adecuada de representar las normas condicionales. Pocas cosas hay tan controvertidas como ésta en la literatura acerca de lógica deóntica. 
(ii) Presupone, también, que los antecedentes de normas condicionales no pueden ser normativos, por la sencilla razón de que carece de sentido, en el lenguaje ordinario, usar un imperativo como antecedente de un condicional. ${ }^{14}$ En consecuencia, los antecedentes de (1) y (2) en R'1 y R'2 son entendidos también como proposiciones normativas $\mathrm{y}$ no como prescripciones.

(iii) Acepta, por lo tanto, algunas limitaciones al encapsulamiento de prescripciones. En concreto y para este caso, las prescripciones no pueden figurar como antecedentes de expresiones condicionales.

(iv) Presupone también que hay alguna forma de definir la relación de consecuencia lógica entre normas distinta de la clásica, es decir, alguna forma de definir consecuencia lógica entre normas que vaya más allá de la verdad. ${ }^{15}$

Cuestiones muy intrincadas de lógica y de filosofía del lenguaje se hallan involucradas en estas asunciones, sin embargo su análisis detallado deberá aguardar a otra ocasión.

\footnotetext{
${ }^{14}$ Sin embargo, Michael Dumett - con el enfoque del cual lo que digo está fundamentalmente de acuerdo- piensa que este rasgo es un rasgo superficial de la gramática de nuestros lenguajes: 'Since the imperatival force is signified in natural language by the mood of the verb, it is necessary to have a rule determining which verbs in a complex sentence are to be put into a imperative mood; and the rule is that only the verb in the main clause, or any co-ordinated main clauses, is affected'. Vd. Michael Dummett, Frege. Philosophy of Language, (Cambridge, Mass.: Harvard University Press, 1981), p. 306 y, en realidad, todo el cap. X'Assertion'.

${ }^{15}$ Puede verse un intento en esta línea, que realiza además algunas restricciones a las expresiones prescriptivas bien formadas y también a las expresiones mixtas (mixtas de aserciones y prescripciones), que reonocen la imposibilidad conceptual de determinados encapsulamientos en José Juan Moreso, Pablo E. Navarro, 'Verdad y eficacia' Theoria 26 (1996): 105-124.
} 